\title{
Exploration on the Integration of Chinese Elements into the Practical Teaching of Illustration Design
}

\author{
Duanwu Shang \\ (City College, Wuhan University of Science and Technology, Wuhan Hubei 430083)
}

Keywords: Chinese Elements; Illustration Design; Practical Teaching

\begin{abstract}
Excellent illustration works should embody certain cultural connotations. At present, many illustration works integrate Chinese elements into them, giving the design works unique traditional Chinese cultural connotations and improving the innovation of the works. Nowadays, with the continuous development of global integration, China's influence on the international community has grown. China's culture and art have also had a profound impact on the development of world art. This paper starts with the practice of illustration design to explore the influence of Chinese elements on illustration design.
\end{abstract}

\section{Connotation of Chinese Elements}

The Chinese element refers to the existence of the traditional Chinese historical and cultural spirit and is a custom that reflects the dignity and national self-esteem of China. Chinese elements are not only a symbol of Chinese culture, but also an important part of global civilization. Chinese elements include traditional Chinese culture, traditional Chinese color expressions, and mythical Chinese ghosts, etc. These are all part of the Chinese element (Fig. 1). For example, in the landscape paintings, there are blank techniques, freehand performance techniques, unique color connotations, and traditional festival characters. China's long history has given birth to a huge Chinese cultural system. Ever since ancient times, Chinese elements have profoundly influenced the world in terms of design, painting, illustration, literature and so on. Chinese elements can be hardly specifically described or defined, but their distinctive style features are distinctly different from Western art elements. The unique artistic atmosphere of Chinese elements is the external expression of Chinese traditional cultural connotations. This is also the charm of Chinese elements.

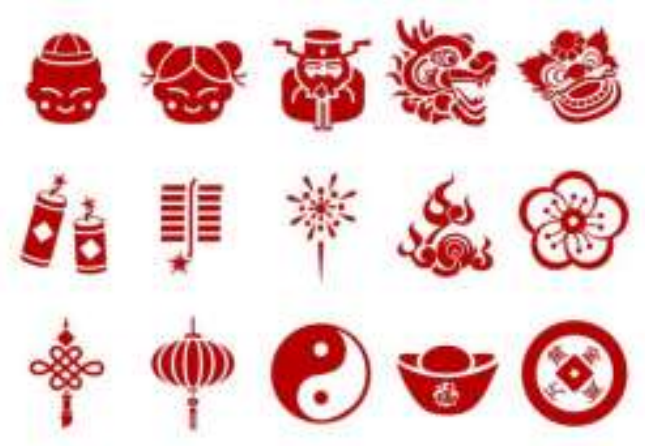

Figure 1. Finite Chinese Elements

\section{Comparison of Chinese and Western Illustration Designs}

In ancient China, the text was obscure and difficult to understand. In order to facilitate readers' reading, several pictures were used as an aid in the book. So, in the past, the illustration got its name. The West has also had illustrations in the past, and the application methods are similar to those in China. Nowadays, the concept of illustration has become more and more general, and there are illustrations in the fields of publishing books, advertising newspapers, and posters that we usually see. The role of illustration is not just to interpret text, but the illustration is also used as an abstract art in many applications. For example, many families use illustrations on the walls, and many curtains also have illustrations. There are obvious differences in culture between China and the West, 
so there are also obvious differences in the daily use of illustrations. For example, Chinese illustrations like to integrate mythological stories (Fig. 2) to add mystery. Western illustrations may use local representative buildings (Fig. 3) to show regional characteristics, and there are obvious differences between Chinese and Western illustrations in the expression of images.

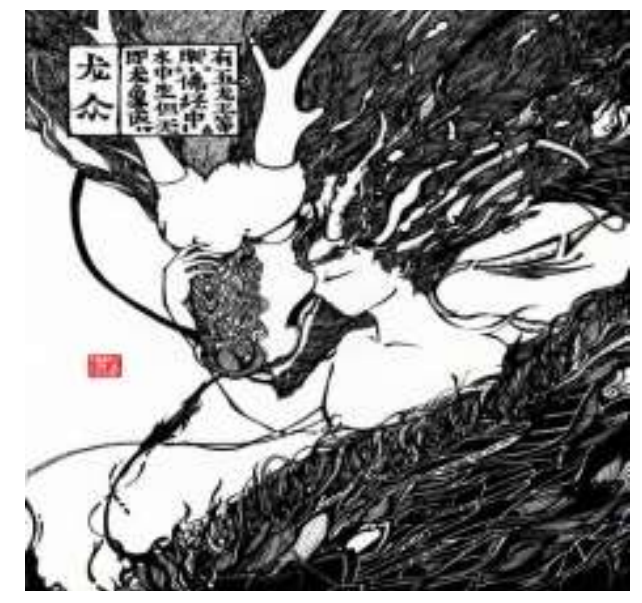

Figure 2. Finite Illustration of Chinese Mythology Story

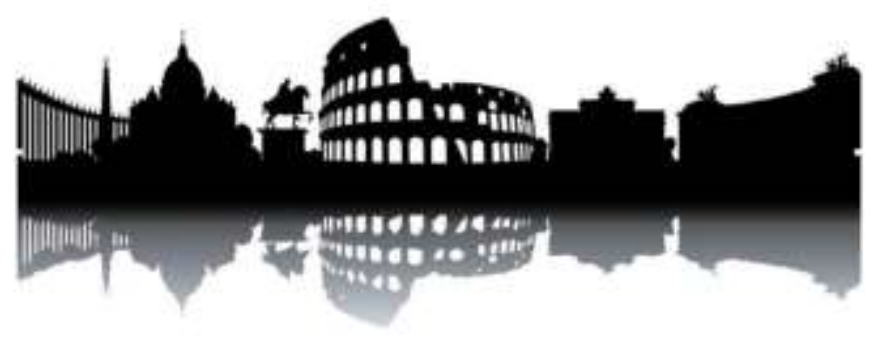

Figure 3. Finite Illustration of Western Buildings

\section{The Innovative Application of Chinese Elements in Teaching Illustrative Design}

If students can understand Chinese elements thoroughly and use them appropriately in illustration design, they will surely add distinctive colors to the work. This not only enhances the cultural connotation of the work, but also plays a role in enriching content and creative expression.

Understand the Connotation and Have Rational Application. Chinese elements can enrich the material of the illustrator classroom teaching content, and should guide students to start from the connotation understanding and rational use when guiding students' creation. Students should understand the purpose of the illustration and the connotation of the work. The works are expressions of inner emotions and souls. They cannot be purposefully added to the use of Chinese elements and deliberately add Chinese elements to the illustration design. This will not only fail to improve the connotation of the work, but may also bring the opposite result, which may make the illustration design look awkward and difficult to understand, and cannot interpret the ideological connotation that the author needs to express. And, it is likely that a joke has occurred because the author's understanding of the Chinese elements is not deep enough. Therefore, you can apply Chinese elements to illustration design, but be natural and don't emphasize it. Today, the classification of illustrations is varied, and illustrations are not limited to abstract or actual expressions, but illustrations are also given other roles. For example, in commercial advertising, illustrators have strong appeal and expressiveness. In art works, illustrations emphasize creativity and artistic imagination. In newspapers and magazines, illustrations have their own realism and expressiveness. As a result, illustrations are increasingly diversified into innovation. The creators must understand the connotation of the elements but are not limited to the expression of a certain 
style, so that the collocation is reasonable.

Focus on Color Matching. In the teaching of illustration design, color matching is very important content. The reasonable collocation and use of colors can allow Chinese elements to better integrate into student illustration works. Teachers should guide students to use colors rationally. Because the illustrations are no longer just black and white with the development of the times, many colors can be used. Students can mix colors according to their needs, modulate the colors they want and then apply them. This will make the pictures vivid and make the traditional pattern and illustration work complement each other perfectly. The meaning of color expression in China is very rich. Generally speaking, the first thing we use in cheerful mood or festival is red or strong color. The unpleasant things are usually black or cool. Therefore, a correct understanding of the environment in which colors are used is very important for the fusion of illustration design and Chinese elements.

Innovative Design based on Traditional Chinese Landscape Painting. Influenced by traditional Chinese landscape paintings, the abstract expressions of illustrations are more and more, and the forms of abstract expressions are becoming more and more abundant. For example, the white space in the landscape painting can leave viewers with a broad imagination and expand and extend the works. If the expression of the inscription can further name the theme of the works, the meaning of the works can be more easily understood. These expression techniques are all influenced by ancient landscape painting in China. At the same time, modern illustration design is not limited to the abstraction of traditional ink landscapes, but also has the abstraction of thinking, such as the use of geometric figures, the combination of lines, the combination of colors and so on. The use of these modern expression techniques can greatly enhance the innovation of illustration design, making the presentation of the work more rich and more diverse. Teachers can make use of landscape painting to demonstrate and explain the techniques and characteristics. Students are required to create corresponding works based on these characteristics and improve students' innovative ability.

Make Full Use of Information Technology for Creation. The development of information technology has provided an effective way for teaching reforms, especially for art disciplines such as illustration courses. Information-based teaching tools have provided great convenience for the development of the curriculum. The use of high-tech means can save students' time and the consumption of creation materials. Currently, drawing software can be used to draw on the art board and project it on the display screen in real time. In this way, it not only solves the problem of ruining the whole picture by a tiny mistake but also helps students to boldly innovate. With the continuous development of technology, the continuous introduction of various graphics software has made it more and more convenient for students to create designs for illustrations. Illustrations are transformed from single drawing drawings to modern information painting. Rich information resources have effectively supplemented the painting content. Reasonable use of mapping software can well integrate Chinese elements into illustration works. Design more original and more Chinese illustrations.

\section{Innovation of Illustration Design Teaching Methods and Ideas}

In the illustration design teaching practice, the author finds that the current illustration design of the students is biased towards the western abstract style. Teachers are also more inclined to westernize illustration design. There are not many uses for the traditional patterns and patterns in the country. However, as a valuable cultural heritage, teachers should require students to improve their cultural connotations and make full use of China's traditional culture.

Integration of Chinese Literatures and Illustration Teaching. The illustrator art curriculum does not exist in isolation, and the multidisciplinary integration teaching is also an effective way to enrich the illustrator classroom teaching. In the teaching of illustration design, the teacher can not only focus on Western color expression and artistic features. In China, there are also many classic designs and legends that can be applied to abstract design teaching. For example, in the Classic Work of Mountains and Rivers, there are many abstract and vivid images (Fig. 4). Among them, the most famous ghost image is the "Big Wicked Beast", which are Dragon Girl, Evil Spirit (Lzdooor), 
Fox Fairy, Yaksha, Horse Face, Ox Head, Erlang, Judge, Qilang, and Tiantian. The vast majority of these images appear in people's eyes in various forms. Each ghost has distinctive characteristics, either milk or umbilicus, or it looks like a fox and nine tails with sounds like baby. All in all, the forms of the ghosts in the Classic Work of Mountains and Rivers have their own characteristics. According to the character of each ghost, they are designed with corresponding colors and visual shapes. For example, the ox head and horse face is cold, so it is usually cold-colored. Nine-tailed foxes are charming and warm colored. Its ghost culture must be said to be extensive and profound. These ghosts appear in some of the mascot illustration designs in our country or some of them use ghost elements. This is enough to show that the influence of traditional Chinese mythological characters and ghost elements on illustration design is very far-reaching. Therefore, in the illustration design teaching cannot blindly biased to a certain direction, we must rationally integrate elements of Chinese and foreign teaching. In order to help students create better, students can be taken to participate in some memorials or museums, allowing students to design mascots with Chinese characteristics.

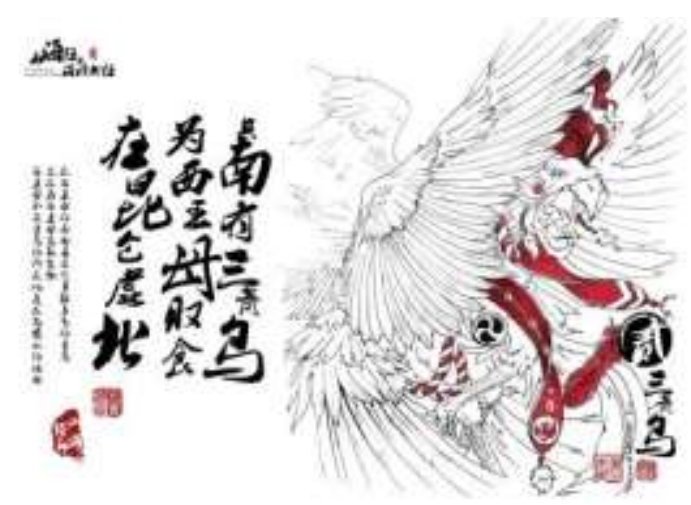

Figure 4. Finite Abstract Animal Images in Classic Work of Mountains and Rivers

Integration of Illustration Teaching and Other Art Teaching. Art is inter-connected, and illustrations may be inspired by other arts. Teachers can take students to museums or other art exhibition halls to study patterns of traditional Chinese objects. The traditional Chinese motifs originated from the painted pottery patterns of the primitive society and have a history of more than 6,000 years. They can be divided into primitive social patterns, classical patterns, folk and folk patterns, ethnic patterns. The ideological content and artistic methods of these patterns mainly reflect the living standards and social politics of the people at that time. This is an ancient socio-political and economic reflection. They all have their own artistic features. In order to apply traditional patterns to their works, students need not only to understand the meaning of their works but also to understand the meaning of the patterns, the meaning of the applications, and which works they are applied to. Only by applying traditional patterns reasonably to your own work can they add color to the pictures. In addition, students can have a look at some of the traditional ways of life or farming methods, which allows them to integrate life into creation and create better illustrations and designs.

\section{Conclusion}

University art teachers should consciously integrate Chinese elements in illustration design teaching, continuously improve students' understanding ability and comprehensive utilization of traditional Chinese cultural connotations, guide students to use Chinese elements rationally in illustration design, and enhance the expressiveness of Chinese elements, innovate design to increase the international cultural influence of Chinese illustration design works. 


\section{Acknowledgement}

This paper is a phased study result of "The Science and Technology Research Project of Hubei Education Department (Project Number: B2017421)"and "The 2016 Research Project of the Education and Teaching Research Project of Qilin Education Research Institute".

\section{References}

[1] Mohammad Talaei; Babak Farhang Moghaddam; Mir Saman Pishvaee; Ali Bozorgi-Amiri; Sepideh Gholamnejad.A robust fuzzy optimization model for carbon-efficient closed-loop supply chain network design problem: a numerical illustration in electronics industry[J].Journal of Cleaner Production, 2016,(06):148-156

[2] Shiva G. Kapsi, Lan D. Castro, Fran X. Muller, Thomas J. Wrzosek.Development of a Design Space for a Unit Operation: Illustration Using Compression-Mix Blending Process for the Manufacture of a Tablet Dosage Form[J].Journal of Pharmaceutical Innovation, 2012, (01):19-29

[3] L. Harivardhan Reddy; Didier Bazile.Drug delivery design for intravenous route with integrated physicochemistry, pharmacokinetics and pharmacodynamics: Illustration with the case of taxane therapeutics[J]. Advanced Drug Delivery Reviews, 2014, (05):34-57

[4] Ameya Anil Kesarkar; N. Selvaganesan; H. Priyadarshan.Novel controller design for plants with relay nonlinearity to reduce amplitude of sustained oscillations: Illustration with a fractional controller[J].IS A Transactions,2015,(07):295-300

[5] Jiachuan Sheng; Jianmin Jiang. Recognition of Chinese artists via windowed and entropy balanced fusion in classification of their authored ink and wash paintings (IWPs) [J].Pattern Recognition, 2014,(02) : 612-622

[6] Sotiria Kogou, Andrei Lucian, Sonia Bellesia, Lucia Burgio, Kate Bailey, Charlotte Brooks, Haida Liang.A holistic multimodal approach to the non-invasive analysis of watercolour paintings [J].Applied Physics A, 2015, (03):999-1014

[7] Soo Mei; Lee @ Jake Lina Lee; Loo Fung Ying; Zaharul Lailiddin bin Saidon.Team-building in the 24 Seasons Drums Education: From Physical Exercise to Music[J]. Procedia - Social and Behavioral Sciences, 2016,(06): 2331-2334

[8] Tang Wei. Analysis on the improvement of intercultural communication ability of students based on applied linguistics [J]. Drama house (the first half of the month),2014,(05):68-70

[9] Fu Ling, Wang Zhenzhen. Traditional pattern and graphic design [J]. China packaging industry, 2014,(11):123-125

[10]Zhang He, Zhou Lijie. New meaning of traditional elements in graphic advertisement creativity [J]. Art education,2015,(02):36-38

[11]Zhang Shengli. Application of national culture in visual communication design [J]. Art education, 2015,(06):84-86 